\title{
Psychological characteristics of adolescents with different levels of digital activity
}

\author{
Irina Luchinkina* and Ekaterina Klimenko \\ Crimean Engineering and Pedagogical University the named of Fevzi Yakubov, 295015, Simferopol, \\ Republic of Crimea, Russian Federation
}

\begin{abstract}
The article discusses the problem of the influence of digital space and the digital environment on the formation of social intelligence and educational motivation of adolescents. The features of the personality included in the digital space are presented and theoretically analysed. Contradictions in the ideas of the "digital personality" are noted. The empirical study revealed significant differences in such parameters as selfconsciousness, self-regulation, empathy, sociability, self-motivation, personal meaning of learning, goal-setting, cognitive and internal motivation of learning among groups of adolescents with low, medium and high levels of digital activity. It was revealed that adolescents with high activity of digital behaviour have low indicators in terms of social intelligence (self-consciousness, self-regulation, empathy, sociability, selfmotivation) and learning motivation (personal meaning of learning, goalsetting, cognitive and internal motivation of learning). Adolescents with medium and low levels of digital behaviour show average and high performance in these parameters. The study showed a significant relationship between low levels of digital activity and cognitive orientation, high digital activity and low self-motivation.
\end{abstract}

\section{Introduction}

In the modern world, the process of digitalisation is gradually spreading not only to the activity sphere of a person but also covers his personality as a whole. The assimilation of digital technologies greatly facilitates the everyday life and life of each person as a whole, but at the same time, it leads to a decrease in the motivation of users to perform physical and cognitive activities. The phenomenon of digitalisation that occurs at the local level reflects the global process of transition of the post-industrial society to the informationoriented society [1-2].

At the same time, Taylor L. Mark notes that despite the variety of technology and information that people receive with its help, one is able to successfully adapt to the digital space and their mental functions. Moreover, according to the researchers, the brain of a modern human continues to evolve (mindfulness is increasing, and consciousness reacts to all signals much faster). As a result, ideation copes with many tasks much more effectively than before [3].

\footnotetext{
*Corresponding author: miss_luchinkina@mail.ru
} 
It is important to note that the digital environment represents an artificial environment that is a regular sequence of computer (network) technologies. The digital environment, as a rule, promotes communication between objects of the physical world by transmitting programs in the form of signals over networks and telecommunications channels. The digital environment exists constantly and independently (without human intervention).

The process of digitalisation covers all existing spheres of human life and manifests itself in the leading types of activities. V. D. Nechaev and E. E. Durneva compare the leading activity (according to D. B. Elkonin) at different stages of growing up with its digital counterpart [4]. The only age that virtual activity does not cover is babyhood $(0-1$ years). However, it is worth noting that today children get acquainted with the digital reality in infancy.

In early childhood (1-3 years), the child first gets acquainted with the digital space. Object-manipulative activity turns into the manipulation of digital devices: modern toys programmed with a binary code, gadgets.

T. Compernolle writes that a preschool child using digital devices may develop a dependence on the color palette, the variety of sounds and game applications. Such addiction, according to the author, leads to a decrease in interest in real space. Based on the research of Compernolle T., the cause of such addictions is the release of dopamine, which occurs when a digital stimulus is presented. Of course, this opinion is interesting and important in the framework of psychophysiological and neuropsychological changes under the influence of technical diversity; however, we note a number of contradictions. The need for a preschooler in a color palette, a variety of sounds and games are explained by the characteristics of the age period.

In addition, it is important to note that digital games for preschoolers, most often, are designed for their current level of development and the zone of proximal development. So, in Google Play and the App Store there are many games for classification by color and shape; games related to spatial thinking, the development of gnosis, etc. The games are specially adapted for preschool children, because they are both entertaining and educational content.

High school age (15-17 years) is characterised by educational and professional activities. It is natural for an adolescent to master the world of professional and economic activity through the Internet and the digital environment. An important role is played by digital technologies that allow one to imagine himself in various roles, play them using virtual reality glasses and devices that simulate real processes of different professions [4].

There is an opinion that a human adapts to the new conditions of a new digital space. The positive effect of adaptation holds the intensive development of cognitive strategies, for example, improvement of the skill of accelerated analysis of incoming signals and the formation of high motivation for certain activities [5-6].

The negative effect consists of a dysregulations of volitional regulation associated with the simplicity of performing a number of tasks and the lack of the need to make efforts to get what is wanted. It can be assumed that dysregulations of volitional regulation can also lead to dysregulations of the motivational sphere in general as its component [7]. For example, students can easily get any information of interest with a few keystrokes, which does not require physical or cognitive load.

One of the features of the digital generation is the awareness that information is easily accessible, which can reduce the motivation to search and memorize it. The absence of the need to record information that is in constant free access is becoming one of the arguments put forward against digital education. It is much easier to find what interests you with a simple keystroke than to analyze and record all the information presented by the teacher. The constant flow of information is also becoming a problem. Naturally, the fact that from childhood the child adapts to the volume and constant change of information is reflected in 
his cognitive processes and strategies. As a result, perception becomes passive, difficulties arise in information processing. One of the phenomena associated with adaptation to a new informational reality is "clip consciousness". This concept indicates that information is perceived quickly and only in fragments. It is difficult for a person to penetrate into its depth, so he reads it superficially, not always taking into account the context and details. From this, a number of researchers conclude that digitalization has a negative impact on cognitive motivation [7].

However, another variant of the impact of digitalisation on the educational and professional motivation of a proprium is also possible. Thus, Wilhelm Wundt in his works writes about the automation of volitional actions, thanks to which it becomes possible to save energy for more significant and complex activities [8]. If we take as a basis the theory identified by Wilhelm Wundt, it can be assumed that the digital environment has a positive effect on increasing of the motivation for more complex cognitive activity of a person.

Brainstorming that takes place in a digital environment leads to success in combining the abilities and knowledge of its users [9]. Different users being experts in particular fields, can use a variety of scientific languages, and thus allow not only to combine research values and standards but also to synthesise methodologies and styles of research. As a result, own culture and skills are formed and developed.

In this regard, by using the capabilities of the digital space one is able to perform more complex mental activities. He doesn't need to waste energy on minor processes that are already automated. The mental activity focuses on a number of cognitive operations (like analysis, synthesis, systematisation, generalisation), creative processing of information and the production of new, previously non-existent [10]. According to T.N. Lombina, there are new phenomena associated with the digitalisation of the cognitive structure of a proprium affecting the process of education. Traditional education of children is hampered by the loss of social skills, Google effect (digital amnesia) and functional illiteracy [10].

The communication of active digital users, according to Rodionova A. S., has also undergone changes. Joint activities involving communication have been replaced by almost automated manipulations with digital devices that do not require speech skills [11]. However, this opinion is also controversial. Generation $\mathrm{Z}$ is famous for its selective attitude to communication and the choice of the social circle. Communication through digital technologies involves new forms of interpersonal interaction which can be more comprehensive than the usual models.

Here, it is appropriate to analyse the problem of the social intelligence of digital users. As in studies of the cognitive and motivational characteristics of digital users, opinions about their social intelligence in the scientific world are divided. A number of researchers note that the digital environment leads to the faster development of empathy, selfconsciousness skills, self-regulation and self-motivation [11-13].

On the other end of the spectrum, according to the researchers, the digital environment leads to blurring of boundaries and, as a result, to difficulties in working on one's personality without auxiliary elements [14-15].

The ambiguity of opinions in the context of studying the digital environment and its impact on a proprium, the lack of methodological apparatus and basic research indicate the need for a theoretical and empirical study of the impact of the digitalisation process on the personal and cognitive sphere of modern human.

Purpose of the study: to analyse the psychological characteristics of adolescents with different levels of digital activity.

\section{Materials and methods}


The study was conducted throughout 2020 in real and virtual spaces. The study involved 100 respondents (of the age of 15-16 years).

At the stage of the ascertaining experiment, the following methods and techniques were used: to determine the level of understanding and perception of the digital environment, focused interviews with adolescents; to identify the level of digital activity of a proprium, the author's research questionnaire was used by I. S. Luchinkina, E. V. Klimenko; to identify the indicators of self-consciousness, self-regulation, empathy, sociability and selfmotivation, the questionnaire "Social Intelligence" was used (the Buss-Durkee test in the modification of G. Rezapkina); to identify the features of the educational motivation of adolescents, the method of studying the motivation of teaching high school students was used (Kalinina N. V., Lukyanova M. I.).

The study of the influence of the digital environment on the personality of an adolescent has identified the problem of the lack of questionnaires and methods that can be used to assess the level of activity of digital behaviour. In this regard, thanks to the analysis of the literature, the author's research questionnaire "Activity of digital behaviour" was developed. It should be noted that the questionnaires describe the types of digital equipment: household appliances (vacuum cleaner, washing machine, microwave oven, refrigerator, etc.); computer equipment (computer, keyboard, laptop, speakers, printer, set-top box, etc.); computing equipment (calculator, scales, computing devices, etc.); transport equipment (passenger cars, buses, minibuses, rail transport, water transport, etc.). This description is given to guide respondents in choosing the appropriate answer option and to classify the respondent's activity in interaction with different types of digital technology.

The respondents were offered statements and questions, the answers to which were the choice of one of the types of digital technology: "I most often use such types of technology as" (to identify motivational and behavioural features); "I am sure that for a high standard of living I need to use such types of technology as" (to identify motivational and behavioural features); "If I see a new model of technology (innovative), then I will most likely understand what it is" (to identify motivational and cognitive features); "It upsets me the most when a breakdown occurs in the following type of technique" (to identify emotive features) and others.

Statistical analysis of the experimental data was performed using the application software "SPSS 23.0" (The Kruskal-Wallis H test, Spearman's rank correlation coefficient, descriptive statistics).

\section{Research results}

For identifying the features of active and inactive behaviour in the digital space, a series of focused interviews among adolescents was conducted. The respondents were asked to tell about their vision of the content of the following categories:

1. Ideas about the digital environment.

2. An active human in a digital environment (who he is, what he does / does not do ...);

3. An inactive person in a digital environment (who he is, what he does / does not do...);

As a result of the analysis of the obtained data, it was found out that the digital environment is an environment consisting of a variety of technology and people who interact with this technology. It is also revealed that the course of interaction with the digital environment depends on the attitudes, expectations, motivation and ideation distinctive feature of the proprium.

If summarise the proposed answers, the idea of an active digital user among adolescents is based on the following thesis: an active digital user has the skills to work with any equipment, understands the mechanisms of its functioning and transfers these skills from one type of equipment to others. 
An inactive digital user, according to the respondents of adolescent age, is prone to a low level of motivation to use technical means for a number of reasons. First, reduced motivation can arise from fear of the new and unknown; second, the peculiarities of the family's (2nd, 3rd generation) attitude to the technical space can have a significant impact on the motivation of teenagers to use the digital environment; third, an important role in the development of active digital behaviour is played by the position of openness to learning new things and strategies for overcoming difficulties.

At the next stage of the research, the sampling was divided by levels of digital activity. There are 3 groups: the respondents with active digital behaviour (36) who are characterised by a high level of motivation, frequent and durational use of technology, as well as a high level of experience with the loss or breakdown of any of the technical equipment; the respondents with a situational level of digital activity (42), who are characterised by a high level of motivation in the use, frequency and duration in the use of certain types of equipment, a feeling of discomfort with the loss or breakdown of any of the technical means; the respondents with a low level of digital activity (22), who demonstrate a low level of interest in the use of digital technology. As a rule, the respondents of this group tend to use the technique only in cases of extreme importance.

The results of the study of the features of social intelligence in adolescents with different levels of digital activity (Figure 1).

The respondents with a low level of digital activity are more tended to high indicators in the parameter of self-consciousness, in contrast to the respondents with a high level of digital activity demonstrating low indicators on the indicated scale respectively $\left(\mathrm{H}_{\mathrm{emp}}<\right.$ 0.05). The respondents with situational digital activity are tended to high and average indicators on the self-consciousness scale $\left(\mathrm{H}_{\mathrm{emp}}<0.05\right)$. Self-consciousness as an element of social intelligence, in the general sense, is an understanding of the characteristics of one's own personality, one's strengths and weaknesses, one's place and role in society.

The indicators for the self-regulation parameter were distributed as follows: the respondents with passive digital behaviour have a high level for this parameter $\left(\mathrm{H}_{\mathrm{emp}}<\right.$ $0.05)$. The respondents with situational digital behaviour have low and high self-regulation indicators $\left(\mathrm{H}_{\mathrm{emp}}<0.05\right)$. The respondents with active digital behaviour showed a low level of self-regulation $\left(\mathrm{H}_{\mathrm{emp}}<0.05\right)$. Self-regulation consists of the following components: the ability to manage one's own behaviour and activities; the ability to be aware of oneself in the current situation and adapt one's behaviour to it.

According to the empathy parameter, high scores were obtained by the respondents with a low level of digital activity $\left(\mathrm{H}_{\mathrm{emp}}<0.05\right)$. The respondents with situational digital behaviour have an average level of empathy $\left(\mathrm{H}_{\mathrm{emp}}<0.05\right)$. The respondents with active digital behaviour showed a low level of empathy $\left(\mathrm{H}_{\mathrm{emp}}<0.05\right)$. Empathy as an element of social intelligence represents compassion for the emotional states of other people and skills of building a communication process by taking into account other people's state.

It is worth noting that the respondents with a low level of digital activity $\left(\mathrm{H}_{\mathrm{emp}}<0.05\right)$ received high results in the sociability parameter. The respondents with situational digital activity have the most pronounced average level of sociability (Hemp $<0.05)$. The respondents with active digital behaviour were found to have a low level for this parameter $\left(\mathrm{H}_{\mathrm{emp}}<0.05\right)$. Sociability as an element of social intelligence is largely responsible not for the number and duration of communication processes but for the ability to maintain a dialogue and the use of labile cognitive strategies in communication.

According to the self-motivation parameter, the respondents with a low level of digital activity $\left(\mathrm{H}_{\mathrm{emp}}<0.05\right)$ received high results. The respondents with situational digital activity have the most pronounced average level of sociability $\left(\mathrm{H}_{\mathrm{emp}}<0.05\right)$. The respondents with active digital behaviour were found to have low and medium levels for this parameter 
$\left(\mathrm{H}_{\mathrm{emp}}<0.05\right)$. Self-motivation is expressed in the ability to maintain performance, building actions and stages for the implementation of a specific goal.

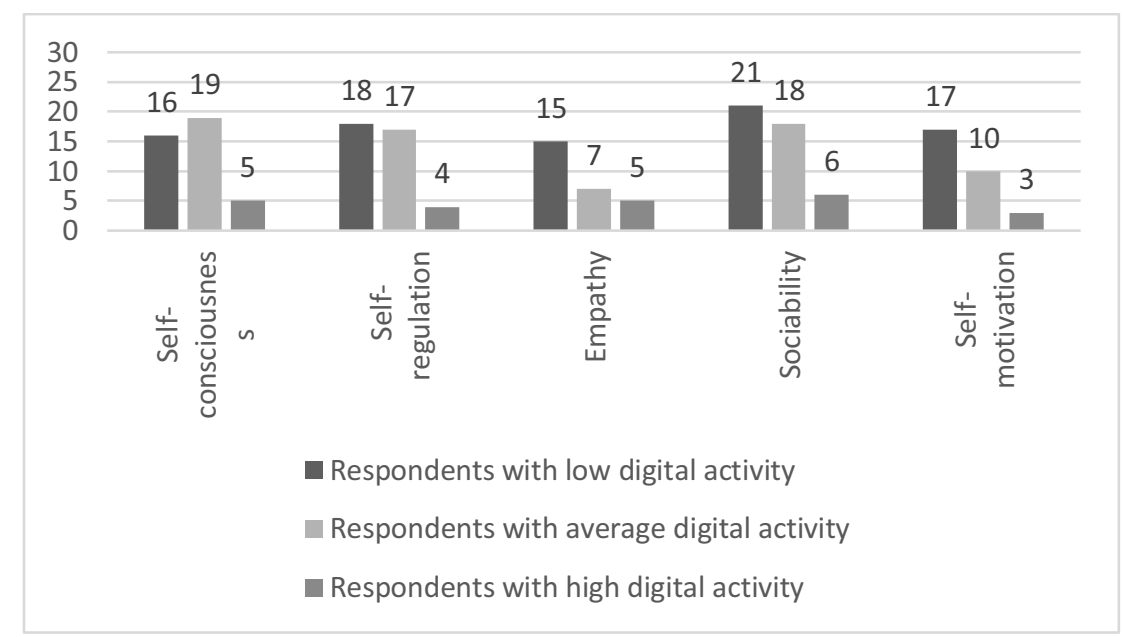

Fig. 1. Results of the study of social intelligence in the sample groups (high performance)

The results of the study of the features of educational motivation in adolescents with different levels of digital activity.

The respondents with a low level of digital behaviour showed a high and very high level of the personal meaning of learning, in contrast to the respondents with active digital behaviour $\left(\mathrm{H}_{\mathrm{emp}}<0.05\right)$. The respondents with situational digital behaviour showed an average and high level of personal meaning invested in learning $\left(\mathrm{H}_{\mathrm{emp}}<0.05\right)$.

The respondents with a passive level of digital behaviour showed average, high and very high goal-setting ability, in contrast to the respondents with active digital behaviour $\left(\mathrm{H}_{\mathrm{emp}}<0.05\right)$. The respondents with situational digital behaviour have a low and average level of goal-setting ability $\left(\mathrm{H}_{\mathrm{emp}}<0.05\right)$.

In the respondents with low digital behaviour, the motive for cognitive activity prevails since the indicators are at a high and very high level (Hemp < 0.05). Among the respondents with situational digital behaviour, the indicators were distributed as follows: $23.8 \%$ of the respondents have motivation for cognitive activity (high level), $60.5 \%$ of the respondents have motivation for cognitive and educational activities (average level), and $15.7 \%$ of the respondents have a predominant motivation for social activity (reduced level) $\left(\mathrm{H}_{\mathrm{emp}}<0.05\right)$.

In the respondents with passive digital behaviour, internal motives of learning activity prevail over external ones $\left(\mathrm{H}_{\mathrm{emp}}<0.05\right)$. The respondents with situational digital behaviour showed a clear predominance of external motives over internal ones $(\mathrm{Hemp}<0.05)$. The respondents with active digital behaviour have a predominant external motivation for learning $\left(\mathrm{H}_{\mathrm{emp}}<0.05\right)$.

The study showed a significant relationship between the levels of digital activity and the parameters of educational motivation and social intelligence: low level of digital activity and focus on cognitive activity $(\rho=0.76)$, high digital activity and low self-motivation $(\rho=0.91)$.

\section{Discussion}


1. It is important to note that the obtained results are typical for the adolescent group of 1516 years. In the studies that conducted in parallel, different results were revealed. For example, in the group of young people (18-25 years old), the respondents with active digital behaviour demonstrate to a greater extent high levels of social intelligence and educational and professional motivation, while the respondents with a low level of digital activity show correspondingly low indicators for these parameters. The same results were obtained during the empirical studies of digital activity in the respondents of mature age (30-40 years old). It is assumed that such differences may be associated with changes in the leading activity and the development of variability of cognitive strategies in the information of perception. However, such assumptions require further experimental confirmation.

2. Confirmation of the previous thesis is provided by some studies aimed at a more mature age. A number of researchers write that the brain and brain activity of people with higher education and a sufficient knowledge base is more labile. It may well be that the digital space will not negatively affect their cognitive, motivational and communicative spheres, but, on the contrary, will strengthen them.

\section{Conclusions}

1. During the focused interview among groups of teenagers, it was revealed that an active digital user is able not only to use digital technology regularly and for a long time, but also to easily introduce new and complex elements into his life.

2. Adolescents with active digital behaviour have low or average indicators in terms of self-consciousness, self-regulation, empathy, sociability, self-motivation, personal meaning of learning, goal-setting, cognitive and internal motivation of learning. In turn, adolescents with passive digital behaviour showed high indicators on these scales.

Adolescents with situational digital behaviour have the most pronounced average indicators in terms of self-regulation, empathy, sociability, and self-motivation. Among the adolescents of this sample, high indicators predominate in the parameter of selfconsciousness, while the parameters of goal-setting ability and motivation for cognitive activity are lowed.

\section{References}

1. T. Compernolle, BrainChains: Discover your brain, to unleash its full potential in a hyperconnected, multitasking world (Compublications, Belgium, 2014).

2. E. Mityagina, N. Dolgopolova, Vestnik NNGU 3 (15), 53-59 (2009).

3. L. Mark Taylor, A Collection of Papers on Self-Study and Institutional Improvement 2 (2), 48-55 (2006).

4. V. Nechaev, E. Durneva, Pedagogika 1, 36-45 (2016).

5. R. Shamionov, Psihologiya social'nogo povedeniya lichnosti (Nauka, Moscow, 2009).

6. A. Verbitsky, Professional'noe obrazovanie. Stolica 7, 10-13 (2016).

7. E. Ilyin, Motivaciya i motivy (Piter, St. Petersburg, 2011).

8. W. Wundt, Vvedenie v psihologiyu (URSS, Moscow, 2007).

9. P. Stefaneas, I.M. Vandoulakis, Metaphilosophy 43 (4), 480-498 (2012).

10. T. Lombina, Obshchestvo: sociologiya, psihologiya, pedagogika 1, 45-40 (2018).

11. A. Rodionova, Perspektivy nauki i obrazovaniya 6, 128-139 (2013).

12. M. Prensky, On the horizon 9 (5), 1-6 (2001). 
13. T. Drabowicz, Correspondence analysis of adolescents in two societies. Computers \& Education 105, 57-67 (2017).

14. Christopher G. Fairburn, Behaviour Research and Therapy 88, 19-25 (2017).

15. P. Norton, Educational Technology 23 (10), 25-28 (1983). 